\title{
El conocimiento profesional de los profesores de ingeniería: una aproximación al problema
}

The professional knowledge of engineering professors: an approach to the problem

Mima Jirón Popova y Carmen Alicia Martínez Rivera

Universidad Distrital Francisco José de Caldas. mjiron@udistrital.edu.co, camartinezr@udistrital.edu.co

\section{Resumen}

En este artículo se presenta un análisis de algunos aspectos relacionados con el conocimiento profesional de los profesores (as) de ingeniería. El análisis se realiza a partir de un cuestionario que respondieron un grupo de profesoras y profesores de la Facultad Tecnológica de la Universidad Distrital Francisco José de Caldas. La información, que fue tratada con ayuda del sofware Atlas.II, permite evidenciar algunos aspectos en relación con el conocimiento profesional y las propuestas de enseñanza.

Palabras clave

Profesor-ingeniero, conocimiento profesional, conocimiento escolar, formación del profesor universitario.

\section{Abstrac}

This artide presents an analysis of some aspects related to the professional knowledge of engineering professors. Analysis is made from a questionnaire filled out by a group of professors of the Technological School in the Universidad Distrital Francisco José de Caldas (Francisco José de Caldas District University). The information was treated through the Atlas.Ti software, which allows showing some aspects connected to the professional knowledge and teaching proposals.

\section{Keywords}

Professor-engineer, professional knowledge, school knowledge, university professor training.

\section{Introducción}

En la universidad, a finales del siglo pasado, cobra vital importancia la necesidad de abordar los problemas relacionados con la didáctica y, en particular, con aquellos concernientes al conocimiento del profesor (a) universitario. La caracterización de este conocimiento ha estado notablemente enriquecida por los aportes de los trabajos de diferentes autores, fundamentalmente desde la propuesta de Shulman (1987). Uno de los aspectos más relevantes, en las diferentes propuestas, es la consideración de que en el proceso de enseñanza los profesores elaboramos un acto creativo, propio de nuestra profesión, que da lugar a un conocimiento particular. Así, por ejemplo, el Conocimiento Didáctico del Contenido $(C D C)$, se constituye en una elaboración producto del proceso de transformación del conocimiento con el propósito de su enseñanza. En general el CDC ha sido descrito como el conocimiento usado para tansformar el contenido de conocimiento en formas más comprensibles para el estudiante. Tendencia en la que parece hay un acuerdo entre diferentes autores, como lo indica la revisión de Park y Oliver (2007). 
La pregunta es qué sabemos acerca de ese conocimiento que han venido elaborando los (as) profesores y, en particular, quienes no han sido formados (as) como profesores (as), como el caso de muchos (as) ingenieros (as). En ese sentido, ¿Cuáles son las posibles relaciones que podríamos plantear entre la mirada como ingenieros (as) y como docentes de ingeniería?. En este escrito pretendemos únicamente realizar una aproximación a esta problemática.

\section{Desarrollo}

Hoy parece que prevalece la tendencia a considerar que el conocimiento de el (la) docente, sobre el proceso de enseñanza y aprendizaje, es fruto de la experiencia docente y no de una formación didáctica (Moreno, 2005). En este sentido, uno de los tema prioritarios en la agenda de discusiones académicas en el ámbito de la formación de ingenieros (as), en Iberoamérica y el Colombia, tiene que ver con papel del Profesor-ingeniero (a), en el proceso de formación de profesionales. Aspecto que fue abordado recientementeii en Colombia, en la XXVII Reunión Nacional de Facultades de Ingeniería: El Profesor de ingeniería, profesional de la formación de ingenieros. Uno de las principales condusiones de dicho evento pone en evidencia que para el Profesor-ingeniero (a) la formación disciplinar debe acompañarse de la experiencia profesional, investigativa y complementada con un riguroso estudio de los saberes, que le permitan el despliegue de la docencia universitaria. Es decir, de la Pedagogía, pero sobre todo, de la Didáctica.

En razón a ello, nos preguntamos, qué aspectos podrían caracterizar el conocimiento de los (as) profesores (as) de ingeniería, teniendo en cuenta sus experiencias de "dlases exitosas"iii. Para aproximarse a la problemática, se solicitó a un grupo de Profesores-ingenieros (as) de la Facultad Tecnológicaiv, de la Universidad Distrital Francisco José de Caldas, que respondieran un Cuestionariov", que busca identificar las ideas que los (as) profesores (as) han elaborado en tomo a su conocimiento profesional y que se proponen como base para su reflexión.

La metodología que siguió el trabajo fue el envío por medio electrónico del cuestionario a los (as) profesores (as) de esa facultad, acompañado de una breve indicación referida a que se esperaba que las respuestas fueran espontáneas. El grupo de Profesores-ingenieros (as) no pertenece al mismo proyecto curricular. La mayoría tienen más de cinco años de experiencia docente, poseen título de maestría en áreas de la tecnología y no cuenta con estudios formales relacionados con la educación. Las dases exitosas presentadas, corresponden al componente profesional del plan aurricular. Las respuestas obtenidas se sistematizaron y categorizaron con ayuda del software Atlas.Ti. Con base en la información recabada, se presentan a continuación las interpretaciones y asociaciones que fueron posibles de consolidar.

Los (as) Profesores-ingenieros (as), asumen como sinónimos el Conocimiento Profesional y la Competencia Profesional (Ver Figura $\mathrm{NO}^{\mathrm{vi}}{ }^{\text {, }}$ líneas continuas). Trátese de Conocimiento 0 Competencia, la Experiencia, entendida como el puente que se establece con una Situación Real, es el factor que determina las Capacidades Profesionales y el Conocimiento Profesional (Ver Figura No1, líneas discontinuas). La Resolución de Problemas, que articula la situación real y la relación teońa-práctica, se configura en el eje central del proceso de enseñanza. Este último, está estrechamente vinculado con actividades de planeación (Preparación de Clases). Es decir, con el establecimiento de Objetivos Previos, Contenidos, Diseño de Actividades de Aprendizaje y de Material de Apoyo y de la Evaluación.

Los (as) Profesores-ingenieros (as) se reconocen a sí mismos como profesionales de la ingeniería, tienen un conocimiento profesional, pero reconoce al profesor como profesional, cuál es conocimiento profesional como profesores

Para los (as) Profesores-ingenieros (as) las bases conceptuales que soportan el dominio temático, son determinantes para el desempeño profesional. Por ello es necesario que dicho 
conocimiento sea actual, es dear, al profesional le corresponde tener pleno conocimiento de los últimos avances que se registran en materia de ciencia y de tecnología. En relación con la Competencia Profesional, algunos de manera puntual consideran que la competencia profesional se mide por la capacidad de resolver problemas en su área de desempeño. Es de anotar, que parece bien diferenciado referirse al profesional como el (la) ingeniero (a) y al (la) docente, que es quien enseña. Por ejemplo, como lo plantea el profesor Luís" cuando afirma: "Creo que un profesor que haya tenido experiencia como profesional (por ejemplo como ingeniero) y se desempeñe como docente, tiene mayor probabilidad de ajustar el desarrollo de su dase a situaciones de "la vida real" que a los planteamientos de los libros, que en la mayoría de los casos solo se quedan a nivel eminentemente teónicos, situación que podría sucederle a un profesional recién egresado..."

La Competencia Docente, por su parte, es considerada como una consecuencia del Conocimiento Profesional. Se podría decir que se supedita a la Competencia Profesional, entendida como Conocimiento Profesional o Competencia Profesional del (la) ingeniero (a). Así, para Pedro, el conocimiento que se requiere para ejercer la docencia universitaria puede ser el mismo del ingeniero (a): "Los conocimientos en un momento dado pueden ser exactamente los mismos [ingeniero (a) o profesor (a) de ingeniería]; varía entonces es en la capacidad de transmitirlo y de enriquecerlo con vivencias y ejemplos'siii.

Otros (as), que posiblemente han reflexionado un poco más el quehacer del profesor (a), diferencian entre la Competencia Profesional como ingenieros (as) y la Competencia Docente. Situación que ilustra Rebecax, al señalar que la Competencia Docente comprende "...Ja capacidad de resolver problemas en su área de desempeño, mientras que, la competencia docente se mide por otros aspectos: ¿soy capaz de guiar al estudiante en su proceso de aprendizaje? ¿Tengo capacidades comunicativas suficientes para facilitar tal proceso? ¿Puedo despertar en los demás el interés por los temas académicos que a mí me interesan? ¿Soy capaz de evaluar los avances de los estudiantes con justicia y equidad? ¿Predominan en mi actuación valores fundamentales como el orden y la responsabilidad? Es daro que el docente también debe tener conocimientos profesionales, pero pienso que éstos mejoran con el tiempo, pues cuando se enseña se aprende. En mi concepto, la distinción entre el conocimiento profesional y el conocimiento docente es muy profunda".

Este último argumento resulta interesante en el sentido de destacar algunos cuestionamientos centrales para el (la) profesor (a) como profesional. El reconocimiento de problemas espećíicos que una comunidad particular, los (as) profesores (as), asumen de manera fundamentada. Estos auestionamientos solo los hace el profesional que se adjudica como objeto de trabajo, los procesos de enseñanza y aprendizaje. Se pude afirmar entonces que los (as) profesores (as) al referirse al Conocimiento Profesional, evocan el conocimiento del ingeniero (Conocimiento Profesional) y no el Conocimiento Profesional Docente.

En lo que respecta al Conocimiento Profesional Docente, se pone de relieve que para los (as) profesores (as), algunos aspectos que lo caracterizan son los siguientes: (1) capacidad de síntesis, (2) competencia comunicativa, (3) creatividad aplicada al diseño de actividades de aprendizaje llamativas, (4) conocimiento de la literatura reciente en el campo del conocimiento respectivo, (5) daridad con respecto a las bases conceptuales que soportan el dominio temático tratado y (6) capacidad de autoevaluación y corrección. Está última entendida como una retrospección con el fin de aprender de los errores y reconsiderar la práctica.

Los (as) profesores (as), reconocen además que hay profesionales que pueden considerarse exitosos en el ámbito de su desempeño profesional, pero que son considerados, por los estudiantes, como profesores poco exitosos. De igual manera, es aceptado el refrán popular que la práctica hace al maestro, por lo que piensan que el desarrollar la docencia por largos años es garante del fioreaimiento de las competencias docentes. 
Es interesante visibilizar que para los (las) Profesores-ingenieros (as) la diferencia entre el Conocimiento Profesional y el Conocimiento Profesional Docente radica en los objetivos. El objetivo del Conocimiento Profesional es la producción, con base en el método científico, de ciencia y tecnología y la solución de problemas y, el objetivo del Conocimiento Profesional Docente es conocer para comunicar. Según Roberto, el "...profesor se acerca de una manera diferente al conocimiento porque su objetivo en relación con el conocimiento de las estructuras químicas busca conocerlas para poder comunicarlas a sus estudiantes, el químico se aproxima a las estructuras utilizando el método cient́fico para tratar de explicar las razones estructurales que garantizan su existencia y que les permiten interactuar con los demás componentes del cosmos". Así, se devela un papel del (la) profesor (a) como trasmisor de un conocimiento producido por otros. Una perspectiva que no permite aludir al profesor como productor de conocimiento. Quizás es por ello, que Roberto para identificar al químico como productor de conocimiento, hace énfasis en el uso del método científica.

Algunas relaciones entre el (la) ingeniero (a) y su docencia

El contexto presentado pone de realce que los (as) Profesores-ingenieros (as), asocian las Competencias Docentes con aspectos tales como: las capacidades didácticas, la planificación de la dase y las estrategias de enseñanza. Las capacidades didácticas, remiten a los estilos de enseñanza, que a su vez son consecuencia de unos presupuestos sobre la pedagogíax, que definen el rol del profesor (a) y del estudiante, en el proceso de enseñanza-aprendizaje. La planificación de la clase son las actividades previas que realiza el (la) Profesor (a)-ingeniero (a) con fines anticipatorios al encuentro formativo. La planificación implica llevar acabo acciones de: Preparación de la Clase, como las nombran, que involucra: (I) el establecimiento de objetivos (competencias), (2) selección de los contenidos necesarios para alcanzar las competencias, (3) preparación de temas, (4) diseño de las actividades de aprendizaje para el logro de las competencias y (5) la selección y/o producción de material de apoyo. Asimismo, las respuestas, ponen de manifiesto la necesidad de reorganizar, sobre la marcha, algunas de las actividades previamente definidas. Reorganización, que puede responder a los intereses propios de los estudiantes, a la necesidad de dedicar más tiempo para el desarrollo de ejercicios de aplicación, al agotamiento que demuestran los estudiantes en determinadas ocasiones, entre otros.

La estrategia que al parecer consideran como la más adecuada, para llevar a cabo el proceso de enseñanza, en la formación de profesionales tecnólogos e ingenieros, es la resolución de problemas (Ver Figura No1), ya que permite poner en equilibro la relación teoría-práctica y acerca a las y los estudiante a la realidad de la actividad que realizaran en su condición de futuros profesionales. Es decir, lo más acorde posible con la realidad que vive el (la) ingeniero (a). Aquí la experiencia del (de la) Profesor (a)-ingeniero (a), en el ámbito profesional, surge nuevamente como uno de los factores de relevancia, para proponer a sus estudiantes un buen número de esas situaciones problemas. En relación con el desempeño de las y los futuros profesionales, es igualmente relevante el rol asignado a las y los estudiantes, en lo que respecta a su participación individual y al trabajo que desarrollan en colectivo (grupo), pues la actividad profesional involucra la interacción con otros. Así, la socialización, emerge como una oportunidad de aprender del otro (a) y de compartir los aprendizajes logrados.

Es necesario además valorar, en su justa medida, que el éxito de las estrategias de enseñanza se asocia con la motivación e interés que pueda despertar el tema en el grupo de estudiantes. Por ello, algunos (as) de los (as) Profesores-ingenieros (as) recurren a lecturas o presentaciones introductorias, que invitan a reflexionar en relación con el contexto que acompaña los temas propuestos. Asimismo, los (as) profesores (as), llaman la atención en relación con la importancia de crear un ambiente de calidez, de conocer el grupo (docente-estudiantes, estudiante-estudiante y estudiantes-docente) y de propidiar situaciones, por ejemplo, que permitan la espontaneidad para compartir una que otra vez alguna anécdota o comentar alguna noticia o suceso, que no necesariamente debe presentarse en relación directa con el tema en desarrollo. 
Tecné, Episteme y Didaxis: TE $\Delta$ No. Extra, 2009

$4^{\circ}$ Congreso Internacional sobre Formación de Profesores de Ciencias

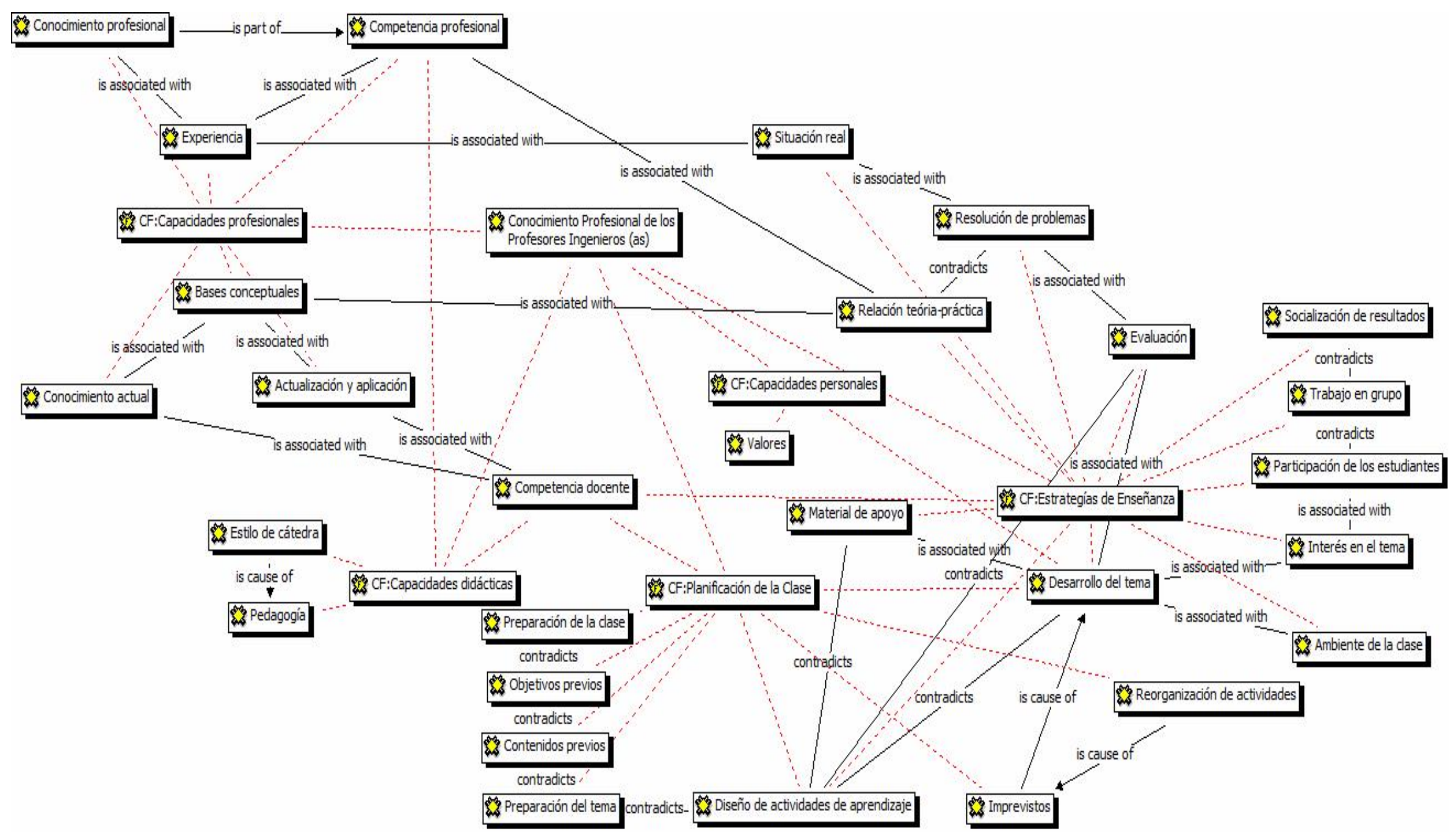

Figura №1. Esquema de relaciones entre aspectos centrales del cuestionario sobre el conocimiento del profesor (a)-ingeniero (a) 
Los aspectos introducidos nos permiten suponer que los (as) ingenieros (as) retoman algunos de los elementos que consideran centrales como profesionales de la ingeniería (planificar, solucionar problemas, relacionar teoría-práctica, etc.) y los trasladan al proceso de enseñanza. Lo aral, pone de manifiesto fundamentalmente la mirada del ingeniero (a), como profesional que proviene de una cultura que se caracteriza por un "., sentido práctico, [ya que los (as) ingenieros (as)] son pragmáticos, su profesión consiste en resolver problemas tecnológicos requeridos por empresas del medio. Son evaluados permanentemente por la comunidad al utilizar aquello que ellos han diseñado" (Millic, 2004:604). Es decir, que para los (as) profesionales ingenieros (as) "la práctica profesional rigurosa es concebida como esencialmente técnica. Su rigor depende del uso de técnicas descriptibles, verificables y replicables, tomadas de la investigación cientúfica y basadas en un conocimiento que sea objetivo, consensual, acumulativo y convergente" (Schön, D., 1996).

El panorama introducido, sugiere interrogantes como: ¿Cuáles son los aspectos que se consideran relevantes de loas (as) profesionales ingenieros (as)?, ¿Qué relaciones son posibles de establecer entre las perspectivas profesionales y como Profesor (a)-ingeniero (a)? Preguntas que requieren el desarrollo de procesos de investigación que enriquezcan el ámbito de conocimiento del (de la) Profesor (a)-ingeniero (a) y, que a la vez, reperautan en los procesos de formación de los futuros profesionales.

Es posible aludir al conocimiento escolar como un conocimiento particular?

Al preguntar a los (as) Profesores (as) ingenieros (as), en relación con el Conocimiento Escolar, la perspectiva apunta a que "... es aquel que se intercambia en el ámbito de la institución escolar. Es un conocimiento particular simplificado y asociado siempre con alcanzar unos objetivos educativos", como afirma Roberto. 0 , aquel que se imparte en el ámbito de una institucional particular la escuela, esto es, en las primeras etapas del proceso educativo (educación primaria 0 secundaria). Supongo, dice Rebeca, "que el conocimiento escolar hace referencia al conocimiento requerido para impartir enseñanza en la escueld"ixi. Esto es, que sí bien se reconocer el Conocimiento Escolar, este se particulariza a la esauela y con ello a los niveles básicos de enseñanza. En ese sentido, parece que el Conocimiento Escolar, no es objeto de conocimiento del nivel de educación superior. Postura que coincide con los hallazgos de Millic (2004), que encuentra que para la enseñanza de la física, en las ingenieńas, el saber sabio difiere muy poco del saber enseñado, la adaptación es tan mínima que incluso muchas veces está fuera de la comprensión de los estudiantes. Otro aspecto que es necesario indagar en nuestros contextos, es aquel que hace referencia las características de las propuestas de Conocimiento Escolar de los profesores universitarios, las diferencias entre quienes tienen formación como profesores y quienes no y, las diferencias entre las culturas académicas de base (ingenieros, químicos, etc) y la cultura de destino (formación de biólogos, químicos, etc).

\section{A modo de reflexiones finales}

Lo expuesto lleva a resaltar las necesidades de generar propuestas de formación de profesores (as), para el nivel de educación superior, que además de atender los aspectos propios de las diferentes áreas profesionales, también incorporen aquellos concernientes a la pedagogía, la didáctica y la investigación educativa. Algunas de las propuestas podrían ser:

> Fortalecer el Conocimiento Profesional Docente, mediante procesos de formación pedagógica y didáctica de los (as) profesores universitarios. En este caso particular de los (as) Profesoresingenieros (as), que como muestra el presente trabajo, parece indicar que es considerado fruto de la experiencia acumulada, en la práctica cotidiana de la docencia universitaria.

$>$ Fortalecer el Conocimiento Profesional Docente, propiciando procesos de reflexión sobre la práctica docente propia. De modo que se asuman de manera explícita y fundamentada los problemas relacionados con la enseñaza y el aprendizaje. En este caso, con la formación de los tecnólogos (as) e ingenieros (as), ya que la totalidad de los (as) encuestados (as) 
manifestaron, de forma verbal, que nunca se había detenido a pensar en su práctica en el sentido en el que fueron invitados por las preguntas incluidas en el cuestionario.

> Fortalecer el Conocimiento Profesional Docente, impulsando procesos de investigación educativa que permitan caracterizar la cultura de base de los (as) ingenieros (as) colombianos (as) y su incidencia en el Conocimiento Profesional Docente, en las concepciones epistemológicas y didácticas, en la elaboración de propuestas de enseñanza y, en el desarrollo de las mismas, etc.

\section{Bibliografía}

Milicic, B. (2004). La cultura profesional como condicionante de la adaptación de los profesores de física universitaria a la enseñanza. España: Universitat de Valencia. Tesis Doctoral.

Moreno, M., (2005). El Pensamientos de los profesores. Evolución y estado actual de las investigaciones. En: Pensamiento $y$ conocimiento de los profesores. Debate $y$ perspectivas internacionales. Perafán, G., Adúriz-Bravo, A. (Compiladores). Bogotá: Universidad Pedagógica Nacional. Editorial Nomos.

Schön, D., (1996). La crisis del conocimiento profesional y la búsqueda de la epistemología de la práctica. En: Pakman, Marcelo (Compilador). Construcciones de la Experiencia Humana. Volumen No.1. Editorial Gedisa. Pp. 183-212.

Shulman, L. S (1987). Knowledge and Teaching. Foundations of the New Reform. Harvard Educational Review, 57 (1). Trad. cast.(2001). Conocimiento y enseñanza. Estudios públicos, 63, 163-196.

PARK Y OLIVER (2007). Revisiting the Conceptualisation of Pedagogical Content Knowledge (PCK): PCK as a Conceptual Tool to Understand Teachers as Professionals. Res Sai Educ.

\footnotetext{
i Traducido de inglés Pedagogical Content Knowledge PCK.

ii Este evento corresponde a la XXVII Reunión Nacional y VI Encuentro Iberoamericano de Facultades de Ingeniería, Cartagena de Indias (Colombia) realizado en Octubre de 2007.

iii Según la elaboración personal de cada profesor, pero en términos de los procesos de enseñanza y aprendizaje.

iv Es importante, para la comprensión de los resultados de las encuestas aplicadas, introducir el contexto en donde se desenvuelve la práctica de los Profesores-ingenieros (as) que participaron de la actividad. El modelo de formación que ofrece la Facultad Tecnológica, se organiza en dos cidos (Jirón, M., Martínez, M., et, al., De Sierra Morena Alta a Candelaria La Nueva. 8 años de la Facultad Tecnológica, Bogota, 2004: 39-40). El Cido Tecnológico, o Primer Cido, con una sólida fundamentación científica y como alternativa de educación superior no terminal y como un cido que propende por una formación con una dara orientación tecnológica evidenciada en la capacidad de solución de problemas reales del entorno productivo, el liderazgo, autoformación, espíritu empresarial, trabajo en equipo, valores personales, manejo del ecosistema y de las herramientas computacionales de sus egresados, que les permita incursionar con éxito en el mercado laboral y en el mundo productivo de las empresas del Distrito Capital y del país. Y, el Cido de Ingeniería, o Segundo Cido, que propende por una formación de ingenieros con un mayor nivel de conocimientos científico y teóricos, que conlleva a fortalecer las competencias y habilidades del tecnólogo en el campo de la investigación \& desarrollo (I\&D) y a su preparación para los niveles postgraduales; objetivos que se logra mediante la profundización en las áreas de ciencias básicas e ingenierías aplicadas.

v Cuestionario propuesto en el marco del Seminario Conocimiento Profesional de los Profesores de Ciencias, del Doctorado Interinstitucional en Educación. Universidad Distrital Francisco José de Caldas. Orientado por la Dra. Carmen Alicia Martínez Rivera.

vi La Figura 1, presenta las relaciones establecidas como resultado de la categorización obtenida con ayuda del software Atlas.Ti (en líneas continuas) y las establecidas adicionalmente en el proceso de análisis (en líneas discontinuas).

vii Los nombres utilizados en el texto son ficticios.

viii También hay quienes señalan que el conocimiento profesional del docente se adquiere en los currículos formalizados, es decir, es propio y particular a los profesionales licenciados.

${ }^{i x}$ Destaquemos que aquí nuevamente la identificación como profesional parece que alude al ingeniero, no al docente.

x Cabe anotar, que son presupuestos sobre la pedagogía de origen empírico, como ya se mencionó los Profesores-ingenieros (as) no cuentan con estudios formales en pedagogía y didáctica.

${ }^{x}$ Las cursivas son nuestras.
} 